\title{
Cavity-Enhanced Room-Temperature Broadband Raman Memory
}

\author{
D. J. Saunders, ${ }^{1, *}$ J. H. D. Munns, ${ }^{1,2}$ T. F. M. Champion, ${ }^{1}$ C. Qiu, ${ }^{1,3}$ K. T. Kaczmarek, ${ }^{1}$ \\ E. Poem, ${ }^{1}$ P. M. Ledingham, ${ }^{1}$ I. A. Walmsley, ${ }^{1}$ and J. Nunn ${ }^{1}$ \\ ${ }^{1}$ Clarendon Laboratory, University of Oxford, Parks Road, Oxford OX1 3PU, United Kingdom \\ ${ }^{2}$ QOLS, Blackett Laboratory, Imperial College London, London SW7 2BW, United Kingdom \\ ${ }^{3}$ Department of Physics, Quantum Institute for Light and Atoms, State Key Laboratory of Precision Spectroscopy, \\ East China Normal University, Shanghai 200062, People's Republic of China
}

(Received 15 October 2015; published 3 March 2016)

\begin{abstract}
Broadband quantum memories hold great promise as multiplexing elements in future photonic quantum information protocols. Alkali-vapor Raman memories combine high-bandwidth storage, on-demand readout, and operation at room temperature without collisional fluorescence noise. However, previous implementations have required large control pulse energies and have suffered from four-wave-mixing noise. Here, we present a Raman memory where the storage interaction is enhanced by a low-finesse birefringent cavity tuned into simultaneous resonance with the signal and control fields, dramatically reducing the energy required to drive the memory. By engineering antiresonance for the anti-Stokes field, we also suppress the four-wave-mixing noise and report the lowest unconditional noise floor yet achieved in a Raman-type warm vapor memory, $(15 \pm 2) \times 10^{-3}$ photons per pulse, with a total efficiency of $(9.5 \pm 0.5) \%$.
\end{abstract}

DOI: 10.1103/PhysRevLett.116.090501

Quantum information technologies such as quantum key distribution and random number generators are beginning to transition into the commercial sphere, where key requirements are the ability to function at high speed, and in nonlaboratory settings. The high carrier frequency of optical signals enables photonic quantum devices to operate noise free at room temperature while offering $\mathrm{GHz}-\mathrm{THz}$ operational bandwidths. However, direct photon-photon interactions are prohibitively weak, which has held back the development of photonic quantum processors. One solution to this problem has been to use probabilistic measurementinduced nonlinearities [1], but the probability of success decreases exponentially with system size, limiting the state of the art to $<10$ photons [2]. The further scaling of photonic devices requires a multiplexing strategy. Quantum memories capable of storing photons and releasing them on demand provide the ability to temporally multiplex a repeat-until-success architecture to achieve a freely scalable photonic quantum information platform operable at room temperature.

There are many types of quantum memory for light under development: electromagnetically induced transparency [3], the full atomic-frequency comb protocol $[4,5]$, gradientecho memories [6,7], and the far-off-resonant Raman memory [8-10]. Each of these protocols has advantages and challenges; see Ref. [11] for a recent review. A helpful metric for temporal multiplexing is the time-bandwidth product $B=\tau \delta$, with $\delta$ being the acceptance bandwidth and $\tau$ the memory lifetime. $B$ is the maximum number of time bins over which a memory can synchronize an input signal. Time-bandwidth products of $B>1000$ enable a dramatic enhancement in the multiphoton rate from parametric photon sources [12], as required to utilize multiphoton interference for computational speedup (e.g., boson sampling [13]). Large time-bandwidth products have been achieved with cold atom memories [14,15], cryogenic rare earth ion memories [16], and room-temperature Raman memories [8,17]. Cavity-enhanced light-matter interactions with atomic ensembles have been demonstrated in many systems-for instance, electromagnetically induced transparency using cold atoms [18] and roomtemperature cavity-enhanced narrow band spin squeezing [19], photon production, and memory operation [20] have been shown. In this Letter we present an implementation of a cavity-enhanced alkali-vapor broadband Raman memory, operating at $75^{\circ} \mathrm{C}$, and show the lowest-noise floor demonstrated in such a room-temperature memory to date.

In a Raman memory, an intense control field induces a two-photon absorption feature far detuned from any atomic resonance. The absorption linewidth of the field-dressed atoms can be made broadband with a pulsed control [17], enabling a large time-bandwidth product even with $\sim \mu \mathrm{s}$ coherence times in a room-temperature vapor [8], and allowing direct interfacing with pulsed heralded photon sources $[21,22]$. Operation with temporally short and fardetuned pulses also removes contamination of the retrieved signals by collision-induced fluorescence [23]. However, large control pulse energies are needed to drive the storage interaction far from resonance, and the control can also drive unwanted four-wave mixing, introducing noise which cannot be filtered out either spectrally or temporally. Fourwave-mixing noise has emerged as the last remaining roadblock to the development of efficient $\Lambda$-type roomtemperature quantum memories [21,24-27]. We solve this 
problem by introducing a new cavity-enhanced Raman memory protocol. We place the atoms inside a low-finesse optical cavity that both enhances the strength of the Raman interaction, reducing the power requirements on the control field, and suppresses four-wave mixing.

To describe how the cavity enhances the memory, we first introduce the standard Raman protocol, which we have developed in cesium vapor at $\sim 70{ }^{\circ} \mathrm{C}[8,21,28-30]$, using the $\Lambda$ system of the Cs $D 2$ line comprising the hyperfine ground states $6 S_{1 / 2}, F=3(|3\rangle), F=4(|1\rangle)$, with a hyperfine splitting of $\Delta_{\mathrm{HF}}=9.2 \mathrm{GHz}$ and the $6 P_{3 / 2}$ excited state manifold $(|2\rangle)$, whose hyperfine structure is unresolved; see Fig. 1(a). We initialize the memory by optically pumping the atoms into $|1\rangle$. Light storage and subsequent retrieval are mediated by the application of bright control pulses with bandwidth $\delta$, time-dependent Rabi frequency $\Omega(t)$, and detuning $\Delta_{s}$ from resonance with the $|3\rangle \leftrightarrow|2\rangle$ transition. This adiabatically couples the signal mode $\hat{s}$ to a delocalized excitation of the storage state $|3\rangle$ described by the spin-wave mode $\hat{b}$, according to the beam-splitter-like Hamiltonian $\mathcal{H}_{\mathrm{BS}} \propto C_{s} \hat{s} \hat{b}^{\dagger}+$ H.c., where the Stokes coupling parameter is given by $C_{s}=\sqrt{W d \gamma} / \Delta_{s}$, with $W=\int|\Omega(t)|^{2} d t$ proportional to the control pulse energy $\mathcal{E}$ and $d$ the resonant optical depth of the $|1\rangle \leftrightarrow|2\rangle$ transition, with linewidth $\gamma$ [34]. The origin of the unwanted four-wave-mixing noise is the coupling of the control field to the ground-excited transition $|1\rangle \leftrightarrow|2\rangle$ with detuning $\Delta_{a}=\Delta_{s}+\Delta_{\mathrm{HF}}$, on which it drives spontaneous anti-Stokes scattering in mode $\hat{a}$, producing anti-Stokes photons and spin-wave excitations in pairs [35]. The interaction is, accordingly, described by a two-mode-squeezing Hamiltonian $\mathcal{H}_{\mathrm{TMS}} \propto C_{a} \hat{a} \hat{b}+$ H.c., with the anti-Stokes coupling given by $C_{a}=C_{s} \Delta_{s} / \Delta_{a}$. If $\Delta_{s} \gtrsim \Delta_{\mathrm{HF}}$, the interactions described by $\mathcal{H}_{\mathrm{BS}}, \mathcal{H}_{\mathrm{TMS}}$ are of comparable strength, and the probability of spontaneously generating a spin-wave excitation approaches that of mapping an incident signal photon into the spin wave. The retrieved fields are then contaminated with thermal noise (half of a two-mode-squeezed state), which destroys the nonclassical statistics of stored single photons [21].

One way to prevent four-wave mixing is to use polarization selection rules such that the control field only couples

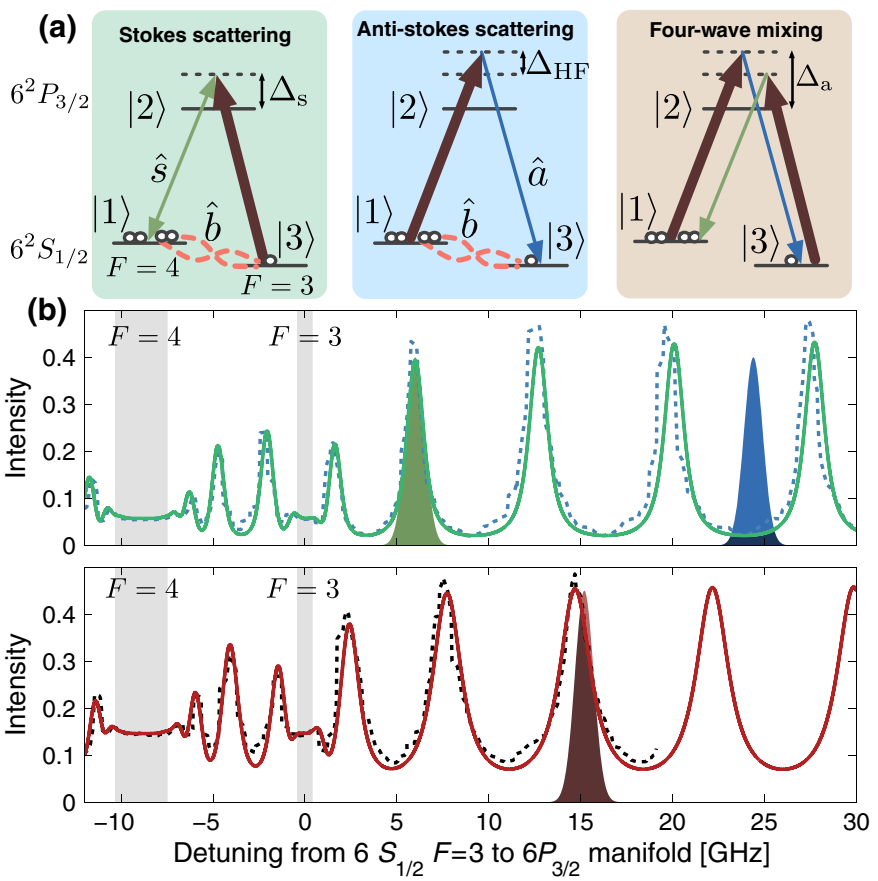

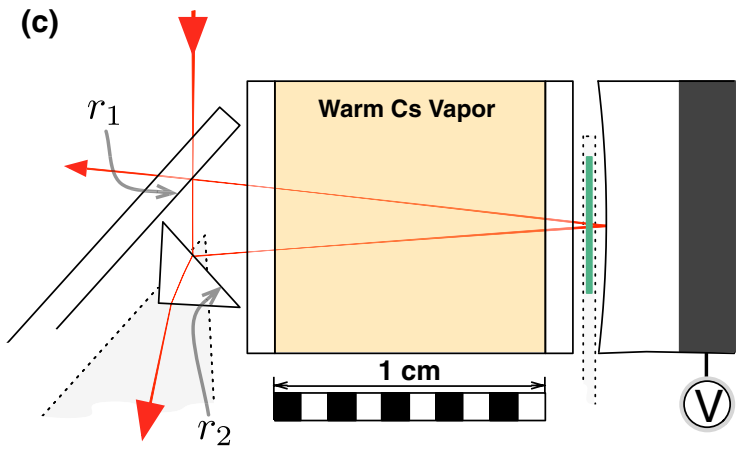

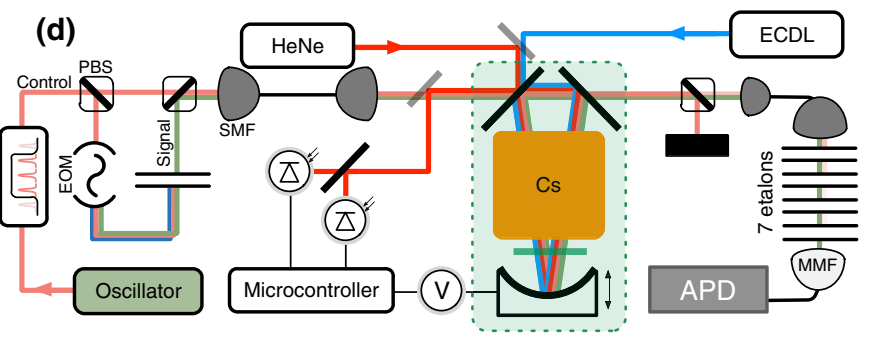

FIG. 1. (a) Energy level diagrams showing the $D 2 \Lambda$ level scheme in Cs. There are two major interactions: one, the Stokes scatteringinvolving the signal field $\hat{s}$ (the green arrow), the control field (the red arrow), and spin wave $\hat{b}$ (the red loop), this is the desired memory interaction; two, anti-Stokes scattering-involving the control field, anti-Stokes mode $\hat{a}$ (the blue arrow), and spin wave $\hat{b}$. The third diagram is the effective noise-process, anti-Stokes scattering followed by Stokes scattering, a four-wave-mixing interaction. (b) Measured frequency response of the atom-filled cavity system. The top is for the signal and anti-Stokes polarization, and the bottom is for the control polarization. The dashed lines are measured responses (see the Supplemental Material [31]) and the solid lines are our theory model using measured values of the cavity parameters [33]. The grey solid areas represent the absorption in the cavity, and they are an indication of our spin polarization, with $\sim 80 \%$ in the ideal $F=4$ state. The green pulse shows the location of the signal and the blue the anti-Stokes, while the red is the control. (c) Scale diagram of the cavity Raman quantum memory. We operate the ring cavity in transmission, where incoupling and outcoupling mirrors have the amplitude reflectivities $r_{1}$ and $r_{2}$, respectively. The curved mirror has $R=40 \mathrm{~mm}$, and the small green rectangle represents the $200 \mu \mathrm{m}$-thick $\mathrm{LiNbO}_{3}$ birefringent crystal, which we tune with temperature to reach the desired cavity conditions. (d) Experimental schematic; for more details see the main text and the Supplemental Material [31] (SMF, single-mode fiber; MMF, multimode fiber; APD, avalanche photodiode; EOM, electro-optic modulator). 
to the $|3\rangle-|2\rangle$ transition. However, for alkali atoms this is not possible due to destructive interference between interaction pathways associated with different intermediate excited states $|2\rangle$ [26]. Four-wave mixing can also be suppressed in dispersive media where it is poorly phase matched $[9,22]$, but the Stokes shift $\Delta_{\mathrm{HF}}$ in alkali vapors is not large enough to introduce a significant phase mismatch. A third approach towards suppressing four-wave mixing is the creation of a second Raman absorption feature at the anti-Stokes frequency [36]. While promising, this method requires the use of an atomic species with three ground states, and it therefore cannot be implemented in ${ }^{133} \mathrm{Cs}$.

The cavity-enhanced Raman memory instead suppresses $\mathcal{H}_{\mathrm{TMS}}$ relative to $\mathcal{H}_{\mathrm{BS}}$ by engineering the density of scattering states so that anti-Stokes scattering into the fundamental cavity mode is forbidden by destructive interference. That is, we arrange for the signal (Stokes) field to coincide with a cavity resonance, while the antiStokes field is antiresonant with the cavity. Setting the freespectral range (FSR) of the cavity accordingly, with $\mathrm{FSR}_{m}=4 \Delta_{\mathrm{HF}} /(2 m+1)$, for an integer $m$, and a roundtrip optical path length $l_{m}=c / \mathrm{FSR}_{m}$, the spontaneous generation of spurious spin-wave excitations by anti-Stokes scattering via $\mathcal{H}_{\mathrm{TMS}}$ is then reduced relative to $\mathcal{H}_{\mathrm{BS}}$ by the ratio of intracavity field amplitudes, parametrized by the noise suppression factor $x=\left(1-\mu_{s}\right) /\left(1+\mu_{a}\right) \approx 0.24$, where $\mu_{s, a, \Omega}$ denotes the cavity-round-trip amplitude losses for the intracavity fields for the Stokes $\left(\mu_{s}=0.6\right)$, antiStokes $\left(\mu_{a}=0.6\right)$, and control $\left(\mu_{\Omega}=0.4\right)$ frequencies. Much greater noise suppression could be achieved with a moderate increase in the cavity quality factor.

With a cavity around the atoms, the acceptance bandwidth of the memory is now limited by the cavity resonance linewidth, so that a broadband memory requires a lowfinesse cavity. However, unlike in single-atom cavity QED [37], where very high cavity quality factors are required to achieve the strong coupling regime and suppress spontaneous emission in any direction, here we require only the suppression of on-axis spontaneous anti-Stokes scattering since off-axis scattering couples to momentum-orthogonal spin-wave modes that are not phase matched [27] and do not contribute noise. Cavity-enhanced ensemble Raman storage is therefore technically less demanding than cavity-QEDbased storage, requiring only low to moderate finesse, which remains compatible with broadband operation $(\delta \sim \mathrm{GHz})$.

The broadest acceptance bandwidth is obtained for a zero-order cavity with $\mathrm{FSR}_{0}=36.8 \mathrm{GHz}$, but to achieve $l_{0}=8.1 \mathrm{~mm}$ would require a monolithic construction. For our demonstration, we instead constructed a second-order cavity with $\mathrm{FSR}_{2}=7.36 \mathrm{GHz}$ and $l_{2}=40.8 \mathrm{~mm}$. We chose a ring geometry instead of a linear one so as not to produce a standing wave inside the cavity since atoms diffusing into the field nodes during the storage time would not interact at retrieval. To incouple the control field, which is linearly polarized orthogonal to the signal and antiStokes modes, we tune the cavity birefringence by heating a thin sliver of $\mathrm{LiNbO}_{3}$ (see Fig. 1) to achieve simultaneous resonance for the signal and the control fields. The cavity length is stabilized with a Hänsch-Couillaud lock [38]: we inject a $\mathrm{HeNe}$ laser into the cavity and feed back on the polarization rotation of the beam by adjusting the cavity length with a piezoelectric actuator (see Fig. 1). A microcontroller (Arduino Due) provides both fast (kHz) [39] and slow (mHz) feedback (see the Supplemental Material [31]).

In addition to suppressing four-wave-mixing (FWM) noise, the double resonance configuration for the control and signal fields reduces the control pulse energy required to drive the memory by a factor of $\sim \mathcal{F}_{s}^{2} \mathcal{F}_{\Omega}^{2} / \pi^{4}$ compared to an equivalent free-space memory, where $\mathcal{F}_{s, \Omega}$ denotes the cavity finesse for the signal and control modes. In our experiment $\mathcal{F}_{s} \sim 7$ and $\mathcal{F}_{\Omega} \sim 4$, combined with the slight imperfect resonance of the control field [see Fig. 1(b)], we obtained a reduction in energy of $\sim 10$. A moderate increase in cavity quality could achieve extremely low operating power.

As can be seen from Fig. 1(b), absorption and dispersion around the atomic resonances significantly modify the cavity mode structure [33]. The Cs vapor pressure depends exponentially on temperature. So, to maintain the resonance condition required for the memory, we developed active temperature stabilization to within $0.1{ }^{\circ} \mathrm{C}$; see Supplemental Material for details [31].

The experimental setup for the memory is shown in Fig. 1(d). The signal and control fields are derived from a modelocked Ti:sapphire oscillator producing pulses with 320 ps duration $(\delta=1.2 \mathrm{GHz})$; details can be found in Ref. [21]. The signal and control pulses are coupled into the same single-mode fiber and directed towards the cavity memory. After the memory we block the control pulses with polarization and spectral filtering, with a rejection of $140 \mathrm{~dB}$ and a transmission for the signal of $\sim 1 \%$, necessary because the control field and the weak-signal field are copropagating and close in frequency. The frequency separation in units of signal bandwidth is $\sim 9$, making frequency filtering challenging. After many air-spaced etalons in series, we detect the transmitted and retrieved signal photons with a Geiger-mode avalanche photodiode (Perkin-Elmer SPCM-AQRH) and a time-to-digital convertor with 81 ps resolution (qutools quTAU). Figure 2(a) shows storage and retrieval with consecutive control pulses separated by $12.5 \mathrm{~ns}$. The input signal average photon number is $0.7 \pm 0.1$ and the memory efficiency is $(9.5 \pm 0.5) \%$, while the noise floor is $\left\langle n^{\text {noise }}\right\rangle=$ $0.015 \pm 0.002$ photons per control pulse in the readout time bin, at the output port of the memory (see the Supplemental Material [31]). This is the lowest noise floor measured in warm vapor Raman memories to date. We also measure the effect of memory efficiency with control pulse energy [see Fig. 2(b)]. We observe a maximum efficiency at $\mathcal{E} \sim 1.5 \mathrm{~nJ}$ before the memory efficiency is decreased due to the dynamical ac-Stark effect. Nevertheless, the measured efficiency agrees with our new theoretical model; see the Supplemental Material [31] and Ref. [40]. This effect can be compensated for by using appropriate pulse shaping $[24,28,41]$. We also measured the 

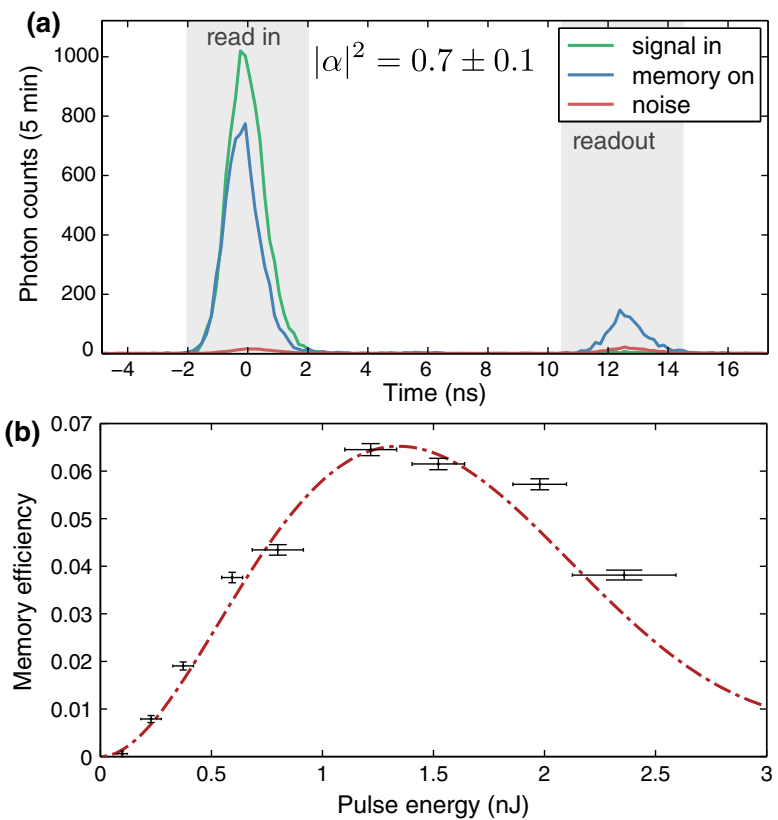

FIG. 2. (a) Arrival time histograms for the signal field emerging from the cavity with the control pulses blocked (signal in), unblocked (memory on) and with the input signal field blocked (noise). This is measured for a coherent state amplitude of $\left|\alpha^{2}\right|=$ $0.7 \pm 0.1$ interacting with the memory. (b) Measured (points) and predicted (line) memory efficiency as a function of the intracavity control pulse energy.

memory lifetime to be $\tau=95 \pm 7 \mathrm{~ns}$, without any magnetic shielding (see the Supplemental Material [31]). This agrees with our previous measurements without shielding, and we expect to easily extend this to $>\mu$ s with magnetic field shielding in the future [8].

The noise floor in our experiment contains contributions from four-wave mixing and also from spontaneous emission. To compare this with the theory, we fit our measurements and extract the FWM $\mathcal{E}^{2}$-scaling component of the noise floor to find $0.006 \pm 0.003$, which agrees well with the theoretical prediction of 0.005 (see the Supplemental Material [31]). Furthermore, a convenient metric to determine whether we have suppressed the noise is the $\mu_{1}$ parameter [4], where $\mu_{1}=\left\langle n^{\text {noise }}\right\rangle / \eta^{\text {tot }}$, a fair metric to compare different devices because it includes both memory efficiency and a noise floor. To determine this accurately, we vary the coherent state amplitude and determine the signal-to-noise ratio (SNR) for each, shown in Fig. 3. By fitting this data, we determine a raw $\mu_{1}$ of $0.17 \pm 0.02$, a considerable improvement compared to free-space Raman memories, for which $\mu_{1}{ }^{\prime} \sim 0.5$ [21]. This is a clear demonstration of noise suppression in a far-off-resonant broadband Raman memory for the first time.

In order to take full advantage of the cavity-induced FWM noise suppression, it will be necessary to remove the remaining sources of noise. This can be achieved by improving the optical pumping efficiency and suppression of counts arising from the pumping laser. The task at hand therefore reduces to a readily solvable engineering challenge.

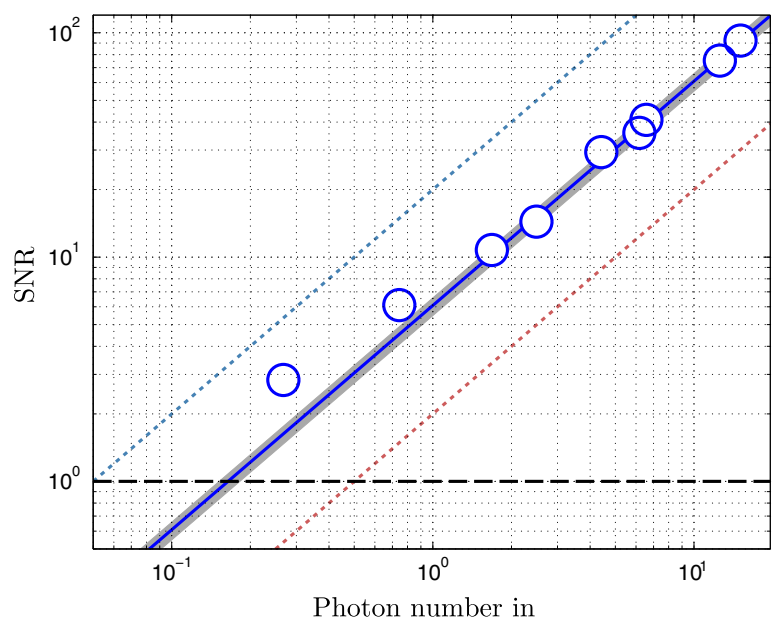

FIG. 3. Measurement of $\mu_{1}$. To measure $\mu_{1}$, we operate the memory with a control pulse energy of $\sim 1.4 \mathrm{~nJ}$, corresponding to our optimal SNR condition. We then measure the signal-to-noise ratio for various input coherent state amplitudes. Where the fits cross the horizontal-black dashed line $(\mathrm{SNR}=1)$ is the value of $\mu_{1}$, with a smaller $\mu_{1}$ desirable. The blue circles are our measured values; error bars are smaller than the marker dimension. The lower-red dashed line represents the state-of-the-art using cavityfree Raman memory experiments [21], with a $\mu_{1}{ }^{\prime} \approx 0.5$. The upper-blue dashed line is the expected performance from our theoretical model, with $\mu_{1}^{\text {Ideal }}=0.005$. We do not reach this ideal noise floor due to experimental imperfections not included in the model (see the Supplemental Material [31]). After fitting our data with $y=x / \mu_{1}$, we extract $\mu_{1}=0.17 \pm 0.02$. The error is calculated using a Monte Carlo simulation.

Once achieved, we can predict the expected performance of a future cavity Raman memory with heralded single photon inputs. Even at the current moderate memory efficiencies, and using spontaneous parametric down-conversion sources which have previously been shown to be compatible with broadband Raman memories [21], interfacing them with our new experiment should enable nonclassical retrieval of single photons [40]. A realistic route to increasing the memory efficiency beyond $10 \%$ is to surpass the current maximum cesium number density caused by light trapping; a process that inhibits optical pumping at high temperatures. This can be avoided by using quenching [42]. Furthermore, we can further increase memory efficiency by compensating for the ac-Stark shift using pulse shaping, or by increasing the finesse of the cavity. Therefore, our new cavity-enhanced Raman memory has laid the ground work for a true roomtemperature quantum memory for broadband heralded single photons in the near future.

We thank Andreas Eckstein for the assistance with the time-to-digital converter, and Benjamin Brecht, Amir Feizpour, and Steve Kolthammer for the insightful discussions. This work was supported by the UK Engineering and Physical Sciences Research Council (EPSRC Grant No. EP/ J000051/1 and Programme Grant No. EP/K034480/1), the Quantum Interfaces, Sensors, and Communication based on the Entanglement Integrating Project (EU IP Q-ESSENCE 
Grant No. 248095), the Air Force Office of Scientific Research: European Office of Aerospace Research and Development (AFOSR EOARD Grant No. FA8655-09-13020), EU IP SIQS (Grant No. 600645), the Royal Society (to J. N.), and EU Marie-Curie Fellowships No. PIIF-GA2013-629229 to D. J. S. and, to E. P., No. PIEF-GA-2013627372. I. A. W. acknowledges a ERC Advanced Grant (MOQUACINO). C. Q. is supported by the China Scholarship Council (CSC Grant No. 201406140039). J. H. D. M is supported by EPSRC via the Controlled Quantum Dynamics CDT under Grant No. EP/G037043/1. The experimental data for this work can be found at ora.ox.ac.uk.

*dylan.saunders@physics.ox.ac.uk

[1] J. L. O'Brien, A. Furusawa, and J. Vuckovic, Nat. Photonics 3, 687 (2009).

[2] X.-C. Yao, T.-X. Wang, P. Xu, H. Lu, G.-S. Pan, X.-H. Bao, C.-Z. Peng, C.-Y. Lu, Y.-A. Chen, and J.-W. Pan, Nat. Photonics 6, 225 (2012).

[3] Y.-H. Chen, M.-J. Lee, I-C. Wang, S. Du, Y.-F. Chen, Y.-C. Chen, and I. A. Yu, Phys. Rev. Lett. 110, 083601 (2013).

[4] M. Gündoğan, P. M. Ledingham, K. Kutluer, M. Mazzera, and H. de Riedmatten, Phys. Rev. Lett. 114, 230501 (2015).

[5] P. Jobez, C. Laplane, N. Timoney, N. Gisin, A. Ferrier, P. Goldner, and M. Afzelius, Phys. Rev. Lett. 114, 230502 (2015).

[6] M. P. Hedges, J. J. Longdell, Y. Li, and M. J. Sellars, Nature (London) 465, 1052 (2010).

[7] M. Hosseini, G. Campbell, B. M. Sparkes, P. K. Lam, and B. C. Buchler, Nat. Phys. 7, 794 (2011).

[8] K. F. Reim, P. Michelberger, K. C. Lee, J. Nunn, N. K. Langford, and I. A. Walmsley, Phys. Rev. Lett. 107, 053603 (2011).

[9] D. G. England, P. J. Bustard, J. Nunn, R. Lausten, and B. J. Sussman, Phys. Rev. Lett. 111, 243601 (2013).

[10] D.-S. Ding, W. Zhang, Z.-Y. Zhou, S. Shi, B.-S. Shi, and G.-C. Guo, Nat. Photonics 9, 332 (2015).

[11] C. Simon, M. Afzelius, J. Appel, A. Boyer de la Giroday, S. J. Dewhurst, N. Gisin, C. Y. Hu, F. Jelezko, S. Kröll, J. H. Müller et al., Eur. Phys. J. D 58, 1 (2010).

[12] J. Nunn, N. K. Langford, W. S. Kolthammer, T. F. M. Champion, M. R. Sprague, P. S. Michelberger, X.-M. Jin, D. G. England, and I. A. Walmsley, Phys. Rev. Lett. 110, 133601 (2013).

[13] S. Aaronson and A. Arkhipov, in Proceedings of the FortyThird Annual ACM Symposium on Theory of Computing, San Jose, CA, 2011 (ACM, New York, 2011), p. 333.

[14] X.-H. Bao, A. Reingruber, P. Dietrich, J. Rui, A. Dück, T. Strassel, L. Li, N.-L. Liu, B. Zhao, and J.-W. Pan, Nat. Phys. 8, 517 (2012).

[15] Y. O. Dudin, L. Li, and A. Kuzmich, Phys. Rev. A 87, 031801(R) (2013).

[16] G. Heinze, C. Hubrich, and T. Halfmann, Phys. Rev. Lett. 111, 033601 (2013).

[17] P. J. Bustard, R. Lausten, D. G. England, and B. J. Sussman, Phys. Rev. Lett. 111, 083901 (2013).

[18] M. Albert, A. Dantan, and M. Drewsen, Nat. Photonics 5, 633 (2011).
[19] G. Vasilakis, H. Shen, K. Jensen, M. Balabas, D. Salart, B. Chen, and E. S. Polzik, Nat. Phys. 11, 389 (2015).

[20] J. Borregaard, M. Zugenmaier, J. Petersen, H. Shen, G. Vasilakis, K. Jensen, E. Polzik, and A. Sørensen, arXiv: 1501.03916.

[21] P. S. Michelberger, T. F. M. Champion, M. R. Sprague, K. T. Kaczmarek, M. Barbieri, X. M. Jin, D. G. England, W. S. Kolthammer, D. J. Saunders, J. Nunn et al., New J. Phys. 17, 043006 (2015).

[22] D. G. England, K. A. G. Fisher, J.-P. W. MacLean, P. J. Bustard, R. Lausten, K. J. Resch, and B. J. Sussman, Phys. Rev. Lett. 114, 053602 (2015).

[23] S. Manz, T. Fernholz, J. Schmiedmayer, and J.-W. Pan, Phys. Rev. A 75, 040101 (2007).

[24] N. B. Phillips, A. V. Gorshkov, and I. Novikova, Phys. Rev. A 78, 023801 (2008).

[25] N. Lauk, C. O'Brien, and M. Fleischhauer, Phys. Rev. A 88, 013823 (2013).

[26] I. Vurgaftman and M. Bashkansky, Phys. Rev. A 87, 063836 (2013).

[27] M. Dabrowski, R. Chrapkiewicz, and W. Wasilewski, Opt. Express 22, 26076 (2014).

[28] K. F. Reim, J. Nunn, V. O. Lorenz, B. J. Sussman, K. C. Lee, N. K. Langford, D. Jaksch, and I. A. Walmsley, Nat. Photonics 4, 218 (2010).

[29] D. G. England, P. S. Michelberger, T. F. M. Champion, K. F. Reim, K. C. Lee, M. R. Sprague, X.-M. Jin, N. K. Langford, W. S. Kolthammer, J. Nunn et al., J. Phys. B 45, 124008 (2012).

[30] M. R. Sprague, P. S. Michelberger, T. F. M. Champion, D. G. England, J. Nunn, X.-M. Jin, W. S. Kolthammer, A. Abdolvand, P. St. J. Russell, and I. A. Walmsley, Nat. Photonics 8, 287 (2014).

[31] See Supplemental Material at http://link.aps.org/ supplemental/10.1103/PhysRevLett.116.090501, which includes Ref. [32], for experimental details, memory lifetime and theoretical description of the noise process.

[32] A. Dantan, A. Bramati, and M. Pinard, Phys. Rev. A 71, 043801 (2005).

[33] J. H. D. Munns, C. Qiu, P. M. Ledingham, I. A. Walmsley, J. Nunn, and D. J. Saunders, Phys. Rev. A 93, 013858 (2016).

[34] J. Nunn, I. A. Walmsley, M. G. Raymer, K. Surmacz, F. C. Waldermann, Z. Wang, and D. Jaksch, Phys. Rev. A 75, 011401(R) (2007).

[35] W. Wasilewski and M. G. Raymer, Phys. Rev. A 73, 063816 (2006).

[36] G. Romanov, C. O'Brien, and I. Novikova, Suppression of the four-wave mixing amplification via Raman absorption, arXiv:1509.08951.

[37] H. P. Specht, C. Nölleke, A. Reiserer, M. Uphoff, E. Figueroa, S. Ritter, and G. Rempe, Nature (London) 473, 190 (2011).

[38] T. W. Hansch and B. Couillaud, Opt. Commun. 35, 441 (1980).

[39] B. Beauregard, http://playground.arduino.cc/Code/PIDLibrary.

[40] J. Nunn, S. E. Thomas, J. H. D. Munns, K. T. Kaczmarek, C. Qiu, A. Feizpour, E. Poem, B. Brecht, D. J. Saunders, P. M. Ledingham et al., arXiv:1601.00157.

[41] A. V. Gorshkov, A. André, M. D. Lukin, and A. S. Sørensen, Phys. Rev. A 76, 033804 (2007).

[42] M. A. Rosenberry, J. P. Reyes, D. Tupa, and T. J. Gay, Phys. Rev. A 75, 023401 (2007). 